\title{
$\beta$-Alanyl-L-Histidine Rescues Cognitive Deficits Caused by Feeding a High Fat Diet in a Transgenic Mouse Model of Alzheimer's Disease
}

\author{
Bruno Herculano, Minami Tamura, Ayaka Ohba, Mayu Shimatani, Natsumaro Kutsuna \\ and Tatsuhiro Hisatsune* \\ Department of Integrated Biosciences, Graduate School of Frontier Sciences, The University of Tokyo, \\ Kashiwa, Japan
}

Accepted 14 September 2012

\begin{abstract}
Our goal in this study was to determine whether or not feeding young (4 months old) Alzheimer's disease model transgenic mice with a high fat diet (HFD), consisting of 32\% fat, is capable of causing cognitive decline and whether treatment with $\beta$-alanyl-L-histidine (carnosine) is capable of reducing these effects. Carnosine is an endogenous antioxidant and antiglycating agent that is abundantly present in the brain and muscle tissues of vertebrates. After 8 weeks of feeding with HFD, we observed a significant decline in the contextual memory in transgenic mice fed with HFD as compared to transgenic mice fed with a normal diet as well as to normal diet-wild type mice. Treatment with carnosine at a dose of $5 \mathrm{mg} /$ day for 6 weeks was effective in preventing cognitive decline, as the transgenic group fed with HFD and treated with carnosine displayed a level of cognition comparable to controls. No differences in senile plaque load were observed between all groups. However, we observed an increase in the expression of RAGE in blood vessels as well as increased microglial activation in the hippocampus of animals fed with HFD, effects that were reversed when treated with carnosine. Given these results, there is a possibility that inflammation and cerebrovascular abnormalities might be the cause of cognitive decline in this model.
\end{abstract}

Keywords: Alzheimer's disease, carnosine, high fat diet, oxidative stress

\section{INTRODUCTION}

Alzheimer's disease (AD) is the most prevalent form of dementia associated with old age in today's society, being present in roughly $80 \%$ of all diagnosed cases of dementia [1, 2]. The onset of its symptoms typically begins with a subtle decline in memory and progresses to global deterioration and adaptive functioning. Recent years have seen the development of novel hypotheses, including a suggestion that has gained considerable strength recently, that there is a

\footnotetext{
*Correspondence to: Tatsuhiro Hisatsune, Department of Integrated Biosciences, The University of Tokyo, Kashiwanoha 5-1-5, Biosciences Bldg., Room 402, Kashiwa, Chiba 277-8562, Japan. Tel.: +81 047136 3632; Fax: +81 047136 3633; E-mail: hisatsune@k.u-tokyo.ac.jp.
}

correlation of lifestyle, obesity, and type 2 diabetes mellitus (T2DM), due to common symptoms observed in these conditions and $\mathrm{AD}$. Cognitive deficits are also observed in patients suffering from T2DM [3], and in recent years much progress has been made in elucidating the link between $\mathrm{AD}$ and T2DM in this regard. Moreover, a recent meta-analysis has also shown a positive correlation between T2DM, obesity, and AD, even though those risk factors seem to be independent of one another [4]. Obesity is a known risk factor for T2DM, and feeding animals with a high fat diet (HFD) is a novel experimental method for reproducing this condition in a laboratory environment [5].

Oxidative stress is one of the earliest events that take place in the pathology observed in AD [6]. This was further evidenced by a study with postmortem 
AD patient samples that has shown the expression of oxidized RNA nucleosides and oxidized amino acid markers in the brains of AD patients [7]. Studies have also demonstrated an accumulation of peroxidized lipids in the brain following the period of feeding with a HFD, which suggests that oxidative stress plays a major role in the observed neuropathology. The accumulation of peroxidized lipids would also impart a certain level of neurological deficits on the offspring of female mice fed with an HFD, implying that the oxidative stress caused by this diet is widespread throughout the body [8]. A recent pivotal study [9] has shown that treatment with antioxidants concomitantly with the administration of donepezil, an acetylcholinesterase inhibitor commonly prescribed for the treatment of early-stage $\mathrm{AD}$, yields a significant improvement in the cognitive function of patients when compared to those treated only with donepezil. The results of this study, taken together with other studies on oxidative stress, have given rise to the hypothesis that treatment with antioxidants is beneficial in the treatment of early-onset AD.

Among the many substances with antioxidant properties available, carnosine has been lauded as a particularly effective one. $\beta$-alanyl-L-histidine (carnosine) is a dipeptide consisting of $\beta$-alanine and histidine, found naturally in the muscle tissue of vertebrates. It is a strong antioxidant substance, being able to scavenge reactive oxygen species (ROS) and products of lipid peroxidation, such as unsaturated aldehydes, and also possessing strong anti-glycating capabilities. Over the past 20 years, studies have shown it to have many important roles in several processes, such as cytosolic buffering, immunomodulation, and neurotransmission [10-12]. Carnosine has been shown to exert a neuroprotective effect when applied before or after an episode of global-brain ischemia [13]. In the same study, it has also been shown to reduce the accumulation of malondialdehyde, a marker of lipid peroxidation, in the brains of animals subjected to an ischemic episode. Pretreatment with carnosine has also been reported as effective in decreasing infarct size, neuronal damage, and ROS levels in models of permanent cerebral ischemia [14-16]. The application of carnosine in vitro has also been reported to be partially effective in protecting neuronal cultures against damage induced by amyloid- $\beta$ (A $\beta)$ [17]. While this particular study refutes the influence of ROS and free radicals on the damage caused by $A \beta$ on neuronal cells, it brings forth the possibility of carnosine exerting a protective effect through its anti-glycating effects. Carnosine is a potent carbonyl scavenger and is capable of chelating transition metal ions, and as such, is considered to be an inhibitor of the formation of advanced glycation endproducts (AGE), a process that chemically alters long-lived proteins $[18,19]$.

Based on this evidence, the objective of this research was to create a new model of obesity-related AD by utilizing HFD. After the establishment of the model, we aimed to evaluate whether the application of carnosine is capable of preventing, even if partially, the cognitive impairments observed in this model of AD. Furthermore, we also aimed to determine possible causes underlying this apparent cognitive decline.

\section{MATERIALS AND METHODS}

\section{Animals}

B6C3-Tg(AßPP swe/PSEN1dE9)85Dbo/J AD model animals were purchased from Jackson Laboratories (Bar Harbor, Maine, USA) and subsequently bred and raised in our laboratory for continuous use in experiments. These animals express the Swedish variation of the phenotype, presenting both a chimeric human AßPP transgene (Mo/HuApp695swe) and human PS1 transgene (missing exon 9) [20].

These genes were transfected separately, and each transgene was driven by its own mouse prion protein $(\mathrm{PrP})$. The use of this promoter ensures expression of the transgenes predominantly into central nervous system neurons.

The animals were interbred with wild type animals of the same lineage (B6C3, Jackson Laboratories, USA) in order to produce wild-type and transgenic animals of a similar background. Animals were initially purchased from Jackson Laboratories (USA) and all subsequent generations were bred at our laboratory. Pairings were carried out starting at 6 weeks of age, and all pairings were composed by one transgenic and one wild-type progenitor.

For the purposes of obtaining a sample consistent with the regular occurrence of $\mathrm{AD}$ in human populations, both male and female animals were used for this study.

Animals were kept in plastic cages in groups of up to 5 individuals depending on the genotype and age of their littermates. The animals were kept in a room maintained at $22^{\circ} \mathrm{C}$ at a constant $12 \mathrm{~h}$ light-dark cycle. All experiments were carried out under the guidelines for animal experimentation enforced by The University of Tokyo. 


\section{Genotyping}

After animals reached the breeding age (roughly 6 weeks of age), they were anesthetized briefly with ethyl-ether and $5 \mathrm{~mm}$ portions of the extremity of the tail were excised and kept ice-cold until the end of the procedure. Animals were kept under observation until the effects of the anesthetic were no longer observed. Tail sections were transferred to tubes containing a lysis buffer consisting of $100 \mathrm{mM}$ Tris, $5 \mathrm{mM}$ EDTA, $200 \mathrm{mM} \mathrm{NaCl}$, and a Proteinase-K solution at a final concentration of $2 \mathrm{mg} / \mathrm{mL}$. The tail sections immersed in this solution was then incubated at $55^{\circ} \mathrm{C}$ overnight.

The cell lysate obtained from this procedure was then subjected to a DNA purification protocol. The final solution containing pure DNA was then subjected to a polymerase chain reaction amplification of the genomic regions containing the A $\beta P P$ and PrP transgenes. The amplified genomic sections were subjected to electrophoresis in a $0.6 \%$ agarose gel. Animals whose samples displayed bands at the densities for both $\mathrm{PrP}$ and A $\beta \mathrm{PP}$ were considered transgenic for the purposes of this experiment.

\section{Feeding with a high fat diet}

Animals used for experimentation with HFD were fed with HFD-32 (CLEA Japan), a mouse feed consisting of $32 \%$ fat, for 8 weeks. The mice had access to these rations ad libitum, and portions were replaced every 2 days to prevent spoiling of the rations and subsequent ingestion of spoiled portions. Control animals were fed ad libitum with the standard rodent feed (MF rations, Oriental Yeast, Japan) utilized for all experimental animals in our laboratory. The components of both feeds are explained in Table 1.

\section{Treatment with carnosine}

Animals used for experimentation with a long-term treatment with carnosine were given a steady dosage of carnosine diluted in de-ionized autoclaved drinking water at the concentration of $1 \mathrm{~g} / \mathrm{L}$. This treatment was started 2 weeks after the initial feeding with HFD and kept until the end of the experiments. Control animals had access to regular de-ionized autoclaved drinking water. All animals had access to drinking water, regular or supplemented with carnosine, ad libitum. Preliminary tests have shown that the presence of carnosine in the drinking water does not cause a significant difference in the amount of daily liquid intake by the mice. Water-diluted carnosine intake was measured to be at
Table 1

Comparative table of the types of feed used for the current experiment. MF feed indicates the standard rodent feed utilized for all animals in the laboratory (Oriental Yeast, Japan), and HFD-32 indicates the high fat diet utilized for the induction of obesity in the present experiment (CLEA, Japan). Fat utilized for the composition of HFD-32 consisted of $80 \%$ of a bovine equivalent of lard and $20 \%$ safflower oil with a high content of oleic acid $(20.5 \mathrm{~g} / 100 \mathrm{~g}$ of ration). HFD-32 contains $7.1 \mathrm{~g}$ of saturated fats, $21.18 \mathrm{~g}$ of monounsaturated fats, and $3.3 \mathrm{~g}$ of polyunsaturated fats per $100 \mathrm{~g}$ of ration. Total sugar content for HFD-32 contains $5.5 \mathrm{~g}$ of cellulose, $8.25 \mathrm{~g}$ of maltodextrin, $6.928 \mathrm{~g}$ of lactose, and $6.75 \mathrm{~g}$ of sucrose per $100 \mathrm{~g}$. All animals were provided with a portion of rations deemed sufficient for ad libitum feeding. HFD-32 rations were discarded and replaced every 2 days as to prevent spoiling

\begin{tabular}{lcc}
\hline & MF Feed & HFD-32 \\
\hline Water $(\mathrm{g} / 100 \mathrm{~g})$ & 7.7 & 6.2 \\
Protein $(\mathrm{g} / 100 \mathrm{~g})$ & 23.6 & 25.5 \\
Fat $(\mathrm{g} / 100 \mathrm{~g})$ & 5.3 & 32 \\
Fiber $(\mathrm{g} / 100 \mathrm{~g})$ & 6.1 & 2.9 \\
Ash $(\mathrm{g} / 100 \mathrm{~g})$ & 2.9 & 4.0 \\
Nitrogen-free extract $(\mathrm{g} / 100 \mathrm{~g})$ & 54.4 & 29.4 \\
Calories $(\mathrm{kcal} / 100 \mathrm{~g})$ & 360 & 507.6 \\
Calories from fat $(\%)$ & 13.25 & 56.7 \\
\hline
\end{tabular}

an average of $5.55 \mathrm{~mL} /$ day, averaging a daily intake of $5 \mathrm{mg}$ of carnosine per mouse, and a $0.177 \mathrm{mg}$ of carnosine per $1 \mathrm{~g}$ of weight at the start of the treatment.

\section{Contextual and cued fear conditioning}

In the last 3 days of feeding with HFD, a fear conditioning experiment was carried out as a means of evaluating the contextual and cued memory of the animals. To carry out this experiment, we utilized a fear conditioning apparatus made by Med Associates Inc. that consists of a metal-acrylic box connected to a video camera, allowing for automated recording and analysis of the experiments. The floor of the box consists of cylindrical metal bars that serve as a means of conducting an electrical current as an aversive stimulus. In order to set the contextual stimulus for the conditioning, the environment was established by constant illumination with a white light and the apparatus was sprayed with a solution of $70 \%$ isopropanol as to set an olfactory contextual stimulus.

Conditioning, contextual memory, and cued memory tests were carried out as previously described [21].

Conditioning was carried out by inserting the animals into the apparatus for $370 \mathrm{~s} .128 \mathrm{~s}$ after insertion, an aural tone was played, followed by a $0.75 \mathrm{~mA}$ electrical shock delivered through the floor of the apparatus. This aural stimulus followed by an electrical shock was repeated for a total of 3 times. The reaction of the animals to this aversive stimulus was recorded by 
the apparatus. $24 \mathrm{~h}$ after the conditioning, we carried out a contextual memory test. This was accomplished by inserting the animals into the apparatus for a total of $512 \mathrm{~s}$, in the exact same environmental conditions as the first day of the experiment, without any further external aversive cues. The fear response of the animals, observable as a "freezing" behavior was evaluated and recorded for analysis. $24 \mathrm{~h}$ following the contextual memory test, the animals were subjected to a cued memory text. This test was performed by changing the environment of the apparatus and observing the freezing reaction to a long aural stimulus. In order to effectively change the environmental cues of the apparatus, we applied a red filter to the illumination of the apparatus, utilized pieces of plastic to make the inside of the apparatus into a triangular form, covered the floor grating with a white piece of plastic and changed the smell of the room with a $70 \%$ ethanol solution. The animals were inserted into this new environment for $640 \mathrm{~s}$, and the same aural stimulus used in the conditioning was played continuously from $128 \mathrm{~s}$ after the animals were inserted until the end of the experiment.

The experimental procedures were captured by the video camera attached to the apparatus, and the resulting video file and raw data calculated by the video analysis software were used for the subsequent analyses. This experiment was carried out by an experimenter blinded to the experimental protocol.

\section{Video data analysis}

All data resulting from the video recordings of the experiments were analyzed by utilizing the VideoFreeze software provided by Med Associates Inc. Data recording was carried out with the following parameters: Motion threshold $=35$, Minimum Freeze Duration $=7$ frames. The video data was recorded at a rate of 15 frames per second. Sampling with these parameters allowed the software to identify a freezing episode when the animal did not display a pattern of motile behavior consistent with the exploration behavior displayed by rodents inserted into novel environments. The video analysis yielded data pertaining to the total of freeze episodes in the total time of the experiments, and this total was divided into equal parts accordingly in order to examine freezing behavior in relation to different stages of the test. In this manner, data for the conditioning experiment was split into 4 segments of $128 \mathrm{~s}$ each, to enable us to discern the freezing behavior related to each of the 3 conditioning cues. Data for the contextual memory experiment was sampled as 4 segments of $128 \mathrm{~s}$ each, in order to account for the fact that the animal acclimatizes to the environment as the experiment progresses. Data for the cued memory experiment was divided into $5 \mathrm{seg}$ ments of $128 \mathrm{~s}$, in order to account for acclimatizing to the continuous aural cue. Data segments up to the first $128 \mathrm{~s}$ were considered as pre-tone data, and the subsequent segments as post-tone.

\section{Collection of blood samples}

$24 \mathrm{~h}$ after the last day of cognitive testing, animals were heated with an incandescent light bulb in order to cause vasodilation and facilitate the collection of blood. After being heated, animals were confined into a restriction tube, and their tail vein was cut with a razor blade at a depth suitable for bleeding. When bleeding occurred at an appropriate rate, approximately $500 \mu \mathrm{L}$ of blood were collected from each animal into microtubes. When bleeding was not sufficient, further cuts, up to a maximum of 3 were made, with caution as not to cause excessive suffering and/or permanent damage to the animals. Minimum blood volume obtained from any given animal was established as $10 \mu \mathrm{L}$, and samples under that volume were properly discarded. Blood samples were then left to rest at room temperature for $1 \mathrm{~h}$ in order to promote coagulation. After $1 \mathrm{~h}$, the tubes were lightly stirred as to detach the coagulated blood from the walls, and centrifuged at $4{ }^{\circ} \mathrm{C}$ at a speed of $5000 \mathrm{rpm}$ for $15 \mathrm{~min}$ in order to separate blood components. After centrifuging, blood serum was collected from the samples and stored at $-20^{\circ} \mathrm{C}$ until analyses were performed. The rest of the blood was appropriately discarded.

\section{Measurement of serum insulin levels}

Insulin contained in the blood serum samples was measured using a commercially available kit for measuring blood insulin in mice (Morinaga, Japan).

\section{Sample processing}

$24 \mathrm{~h}$ after the last day of cognitive testing, animals were sacrificed and perfused transcardially with a $4 \%$ paraformaldehyde solution for the fixation of the brain tissue. Brains samples were then obtained and incubated in the same $4 \%$ paraformaldehyde solution for $24 \mathrm{~h}$. After this primary incubation, samples were incubated in a $30 \%$ sucrose/PBS solution for 3 days, before being embedded in Tissue-Tek OCT compound (Sakura Finetek, Japan) and frozen at $-80^{\circ} \mathrm{C}$. Samples were then sliced at the thickness of $40 \mu \mathrm{m}$ in a Cryostat 
(Microm, Germany). The acquisition of brain slices started from the Bregma, and continued until roughly 48 slices containing the hippocampus were obtained. Slices were then submerged in a cryoprotectant solution and stored in $\mathrm{a}-30^{\circ} \mathrm{C}$ freezer.

\section{Histochemical staining}

Samples were washed 3 times in TBS and incubated with X-34, a dye for staining senile plaques for $30 \mathrm{~min}$.

X-34 (1,4-bis(3-carboxy-4-hydroxyphenylethyl)benzene) is a highly fluorescent dye derived from Congo red that binds specifically to amyloid aggregates and allows for a detailed fluorescent visualization of the localization and distribution of senile plaques [22].

The X-34 solution was synthesized in our laboratory from tetraethyl $\mathrm{p}$-xylylenediphosphonate and 5-formylsalicylic acid. After staining with X-34, samples were briefly washed in TBS and incubated for $2 \mathrm{~min}$ in a $0.2 \mathrm{~g} \% \mathrm{NaOH} / 80 \%$ ethanol solution. Samples were then washed again with TBS for $5 \mathrm{~min}$ and incubated with TOTO-3 iodide (Molecular Probes, USA) in TBS for $5 \mathrm{~min}$, in order to stain cell nuclei. Samples were washed again with TBS for $5 \mathrm{~min}$. Samples were then mounted on microscope slides and visualized using a fluorescence microscope equipped with an ultraviolet laser and an ultraviolet filter (Olympus, Japan). Images were then obtained utilizing a digital camera coupled to the microscope.

\section{Immunohistochemical staining}

Samples were washed 3 times in TBS containing $0.1 \%$ Triton-X (0.1\% TBS-X), then blocked with $3 \%$ normal donkey serum diluted in $0.1 \%$ TBS-X for $30 \mathrm{~min}$ at room temperature. Following the blocking procedure, samples were then incubated with specific antibodies for the receptor for AGE (RAGE) (rabbit-derived; $1: 100$ dilution; Abcam Japan), cluster of differentiation 31 (CD-31) (mouse-derived; $1: 50$ dilution; BD Pharmingen Japan), glial fibrillary acidic protein (GFAP) (mouse-derived; 1:500 dilution; Sigma Japan), ionized calcium binding adaptor molecule 1 (Iba1) (rabbit-derived; 1:1000 dilution; Wako Japan), and acrolein (mouse-derived; 1:100 dilution; NOF Japan) overnight at $4{ }^{\circ} \mathrm{C}$. Samples were then washed 3 more times with $0.1 \%$ TBS-X and incubated with specific secondary antibodies conjugated with Alexa-488 (donkey-derived; anti-rabbit; 1 : 1000; Molecular Probes) and Cy5 (donkey-derived; antimouse; 1:250; Jackson Immuno Research) for $2 \mathrm{~h}$ protected from light at room temperature. After incubation, samples were washed with TBS once, and then incubated with 4'6-diamidino-2-phenylindole (DAPI) ( $1: 10000$ dilution; Sigma) in TBS for $5 \mathrm{~min}$. After one additional washing with TBS to remove excess DAPI, samples were mounted on microscope slides and visualized using a fluorescence microscope equipped with an ultraviolet laser and an ultraviolet filter (Olympus, Japan). Images were then obtained utilizing a digital camera coupled to the microscope.

\section{Image processing}

Images obtained through microscopy and X-34 staining were analyzed with the ImageJ software. A custom plugin was utilized in order to automatically establish the overall fluorescence of each imagine and calculate the percentage of the image covered by senile plaques (X-34 fluorescent spots). The plugin was used for automatic analysis, and the regions of interest (ROI) utilized for the analysis of each image were then inspected manually by an experimenter in a debug mode in order to properly eliminate false positives and negatives.

\section{Statistical analyses}

All data were analyzed for significant differences utilizing a non-paired two-tailed Student's $t$ test. All differences were considered statistically significant when $p<0.05$.

\section{RESULTS}

One of the objectives of this study was to establish a reliable model that encompasses risk factors suspected to be responsible for AD. As such, we sought to check whether a short term feeding with a HFD was capable of evoking cognitive decline in $\mathrm{AD}$ transgenic mice, and concomitantly we sought to determine whether a short term treatment with carnosine was effective in countering this decline. In this model, we used 4 month old transgenic animals, and fed them with a HFD diet for 2 months, performing cognitive test as they reached 6 months of age. Treatment with carnosine was carried out for 6 weeks, starting 2 weeks after the feeding with HFD began. The groups and timeline of this experiment are delineated in Fig. 1A. All groups in the experiment were treated and evaluated at the same time, but for ease of understanding we shall explain the experiments progressively through a logical progression of the observed results. 

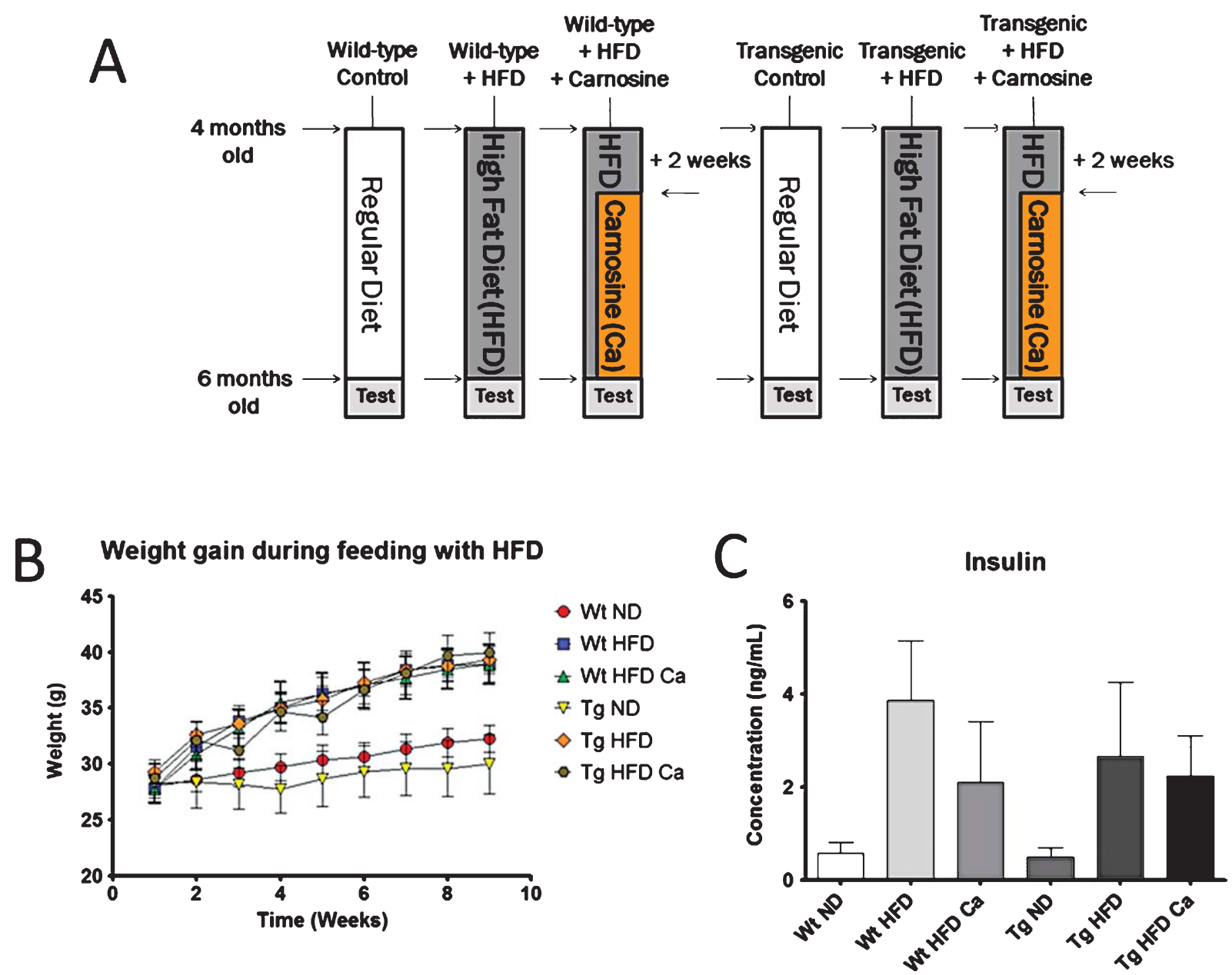

Fig. 1. Experimental paradigm: A) Schematic of the experimental protocol utilized for the current experiment. Wild-type and transgenic animals were subject to a regulated diet for 2 months before cognitive testing. Control animals were fed with MF feed (Oriental Yeast, Japan; refer to Table 1) ad libitum for the whole duration of the experiment. Animals fed with a high fat diet (HFD-32; CLEA, Japan; refer to Table 1) for a period of 2 months, starting at 4 months of age. Rations were discarded and replaced every 2 days as to prevent spoiling. Rations were given in a quantity that allowed animals to feed ad libitum. 2 weeks after the beginning of the feeding, animals treated with carnosine were given carnosine at a dose of $5 \mathrm{mg} / \mathrm{day}$, administered through their drinking water. The treatment continued until the end of the experiment. 2 months after the start of the experiment, animals were subjected to a contextual fear conditioning test. B) Weight of the animals throughout the experiment. Animals fed with HFD displayed markedly increased weight at the end of the experiment. Consumption of carnosine did not influence weight gain on any of the groups tested. C) Serum insulin levels of animals utilized in the experiments. $24 \mathrm{~h}$ after the end of the cognitive experiments animals here heated to provoke vasodilation, immobilized, and had minor cuts made on their tails in order to partially cut the tail vein and cause bleeding. Blood samples were centrifuged, and only the serum was used for the analysis. Analysis was carried out utilizing a mouse-specific commercially available serum insulin assay kit. Bars: SEM; Wt, wild-type; Tg, transgenic; HFD, high fat diet; Ca, carnosine; ND, normal diet. $n \geq 3$ for all groups.

First we set to determine whether feeding with HFD for a relatively short period ( 8 weeks, or 2 months approximately) would impart in those animals a degree of obesity and the related metabolic syndrome sufficient for evoking AD-like symptoms. As a result, we observed that feeding with an HFD for a period of 8 weeks was enough to evoke a significant increase in body weight, which was not capable of being reverted by treatment with carnosine (Fig. 1B).
We measured the insulin levels of blood samples collected from the animals, in order to assess whether feeding with HFD would cause hyperinsulinemia, a condition that indicates the occurrence of a metabolic syndrome. As a result, feeding with HFD seemed to cause an increased level of insulin in the blood of the animals, and treatment with carnosine did not significantly alter the levels of blood insulin (Fig. 1C). 

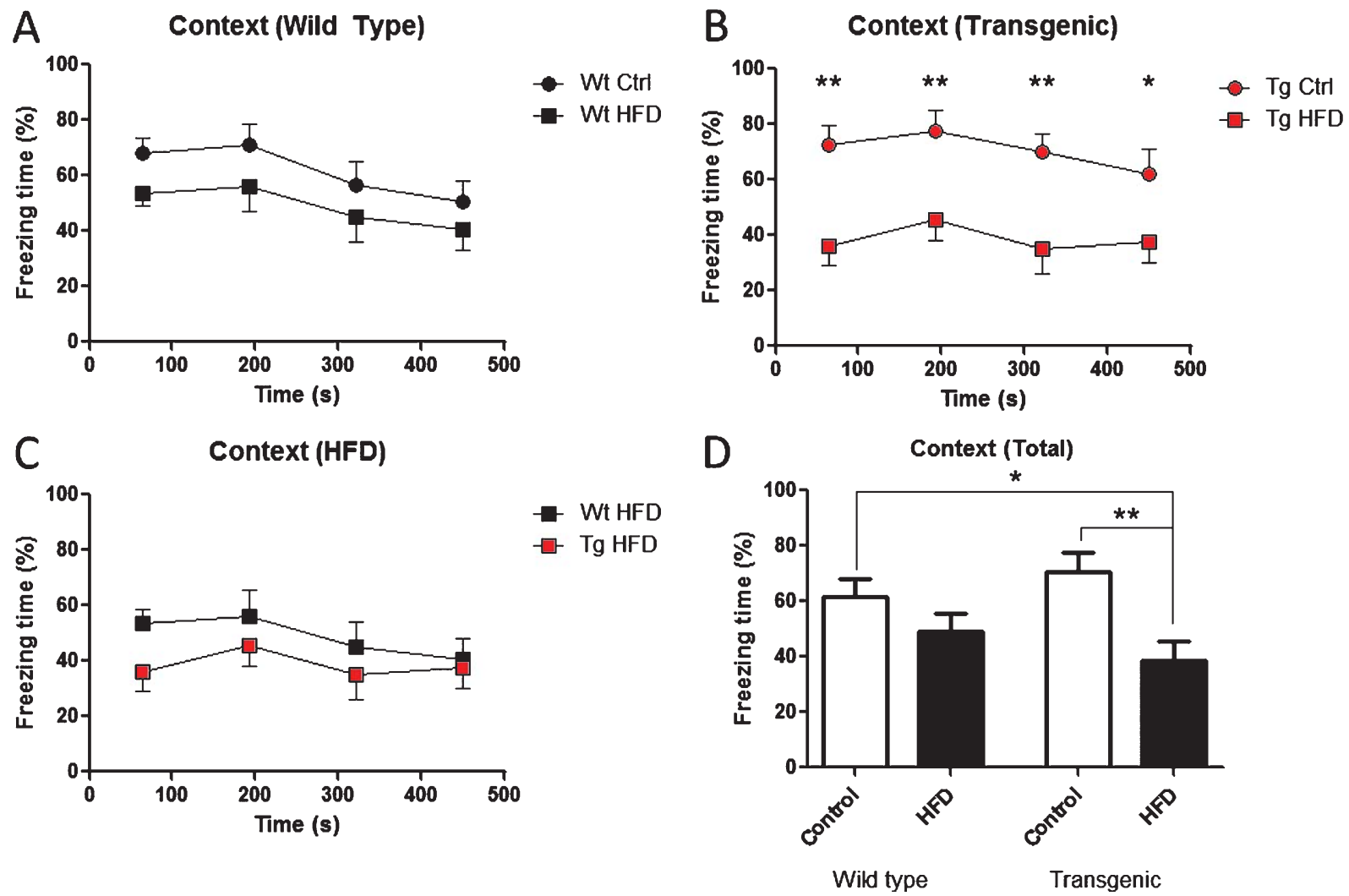

Fig. 2. Contextual memory impairment in transgenic young mice fed with a high fat diet (HFD). Young wild type and transgenic mice (6 months old) were fed with a HFD for 2 months, and were then subjected to the fear conditioning test in order to assess whether feeding with a HFD is capable of evoking cognitive impairments. A) Comparison between the wild-type control group and the wild-type group fed with a HFD. B) Comparison between the transgenic control group and the transgenic group fed with a HFD. C) Comparison between the wild-type and transgenic groups fed with a HFD. D) Comparison of the total freezing time observed it the experiment in all groups. Bars: SEM; Wt, wild-type; $\mathrm{Tg}$, transgenic; HFD, high fat diet. $n=11$ for the wild-type group, $n=10$ for the wild-type group fed with a HFD, and $n=13$ for the transgenic groups; ${ }^{*} p<0.05,{ }^{* *} p<0.01$.

This treatment was also capable of causing cognitive impairments in the contextual memory $(p<0.05$ for the transgenic group fed with a HFD when compared to the wild-type group fed with a regular diet and $p<0.01$ when compared to the transgenic group fed with a regular diet) (Fig. 2). That degree of cognitive impairment was considered similar to that observed as a symptom of the onset of AD in aged animals, utilized in previous experiments in our laboratory.

Transgenic animals fed with a HFD also presented a slight impairment on their cued memory $(p<0.05$ for the transgenic group fed with a HFD when compared to the wild-type group fed with a regular diet) (Fig. 3), an effect that was not observed in aged animals (data not shown). This data suggests that feeding with a HFD imparts a wider functional impairment than the one caused solely by the genetic background of the transgenic animals.
After evaluating the extent of the cognitive deficits elicited by feeding with a HFD, we arranged to examine whether a short-term treatment (6 weeks) with carnosine, an antioxidant compound found naturally in the muscle and brain tissue of mammals, was capable of preventing the cognitive decline caused by feeding with an HFD in transgenic AD model animals.

Treatment with carnosine, at a dose of $5 \mathrm{mg} /$ day, administered through the drinking water proved effective in preventing the cognitive decline observed in the animals fed with HFD. This effect was seen both in their contextual (Fig. 4) and cued memory (Fig. 5). The treatment was able to maintain cognition and memory to a level statistically comparable to the control group, further attesting to its effectiveness.

Following the analysis of blood samples, brain samples were analyzed for the presence of senile plaques that would indicate the onset of $\mathrm{AD}$ at the histological 
A Cue (Wild Type)
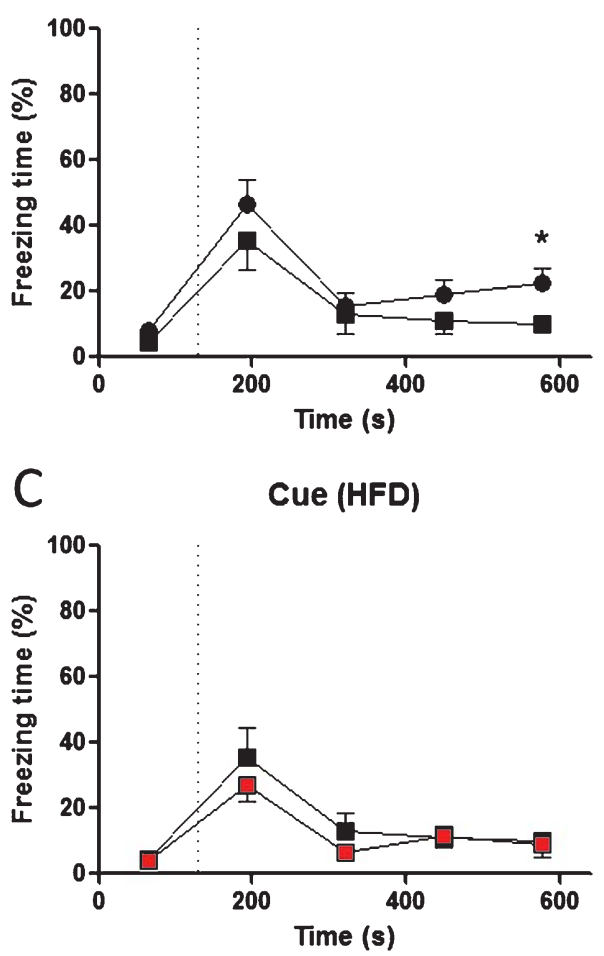

B Cue (Transgenic)
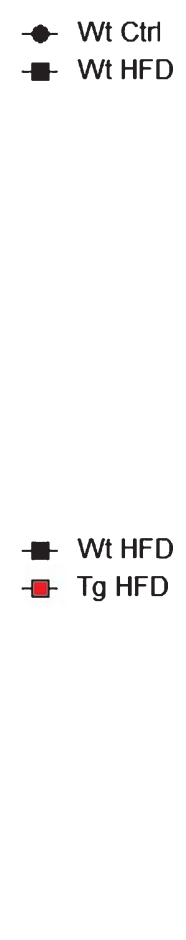

D

Cue (Total)
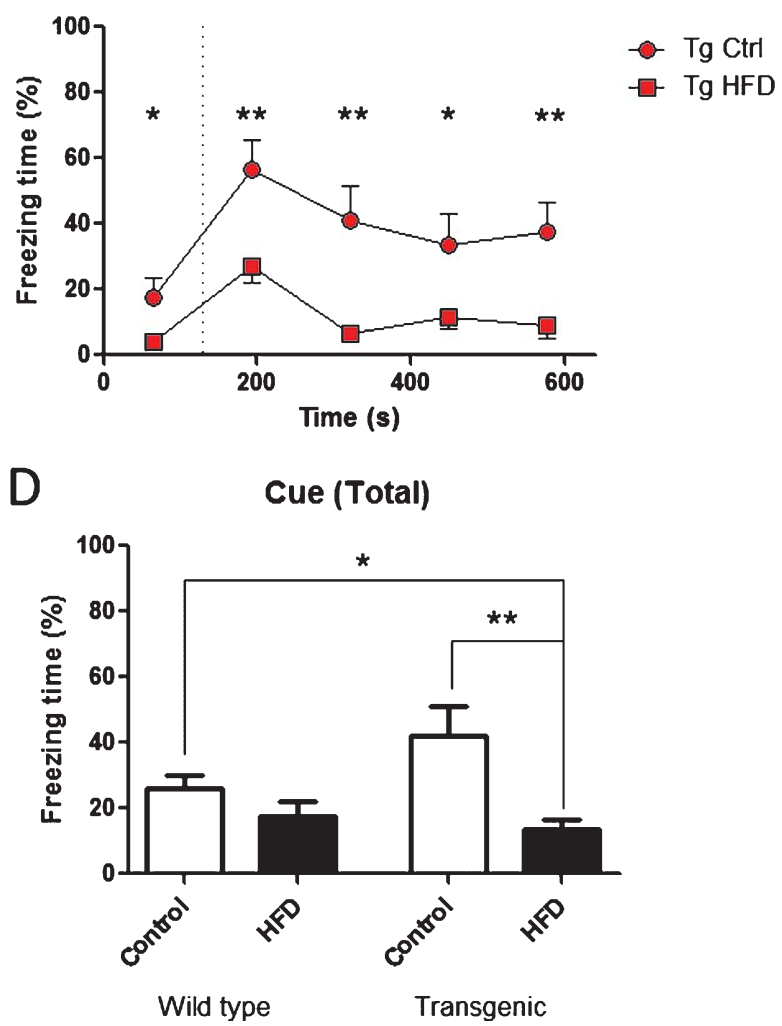

Fig. 3. Cued memory impairment in transgenic young mice fed with a high fat diet (HFD). Young wild type and transgenic mice (6 months old) were fed with a HFD for 2 months, and were then subjected to the fear conditioning test in order to assess whether feeding with a HFD is capable of evoking cognitive impairments. A) Comparison between the wild-type control group and the wild-type group fed with a HFD. B) Comparison between the transgenic control group and the transgenic group fed with a HFD. C) Comparison between the wild-type and transgenic groups fed with a HFD. D) Comparison of the total freezing time observed it the experiment in all groups. For the purposes of this experiment, only time points after the aural cue were used to calculate the total freezing time. Bars: SEM; Wt, wild-type; Tg, transgenic; HFD, high fat diet. $n=11$ for the wild-type group, $n=10$ for the wild-type group fed with a HFD, and $n=13$ for the transgenic groups; ${ }^{*} p<0.05,{ }^{* *} p<0.01$.

and cellular level. As a result, we could observe no senile plaques in the hippocampus of wild-type animals (data not shown), fed with a regular diet or HFD, and treating these animals with carnosine did not cause any changes on the formation of senile plaques on the hippocampus (data not shown). Interestingly, while we did not observe any significant cognitive deficits in the young transgenic animals fed with a normal diet, we did observe the formation of senile plaques in their hippocampi (Fig. 6A). This fact indicates that the presence of senile plaques per se might not be an effective indicator of cognitive deficits, at least in this particular animal model of AD. Treatment with carnosine during the time of feeding with a HFD was not capable of preventing the formation of senile plaques in the hippocampus of transgenic animals (Fig. 6A). From that point, we recurred to software analysis to determine whether there were any significant differences in the formation of senile plaques among all groups. As a result, we found no difference in the area covered by senile plaques in the hippocampus of animals from all groups (Fig. 6B). Interestingly, treatment with carnosine did not seem to be effective in countering the formation of senile plaques, indicating that it exerts its protective or healing effects through means that are independent of the amyloidogenic pathway.

Following these experiments, we next aimed to determine which changes might be responsible for this evident decline in cognition. Given the reported antiglycating properties of carnosine, we postulated that an increase in glycated proteins caused by feeding with HFD would reflect in an increase in the expression of RAGE in the brain. Thus, we proceeded to stain 

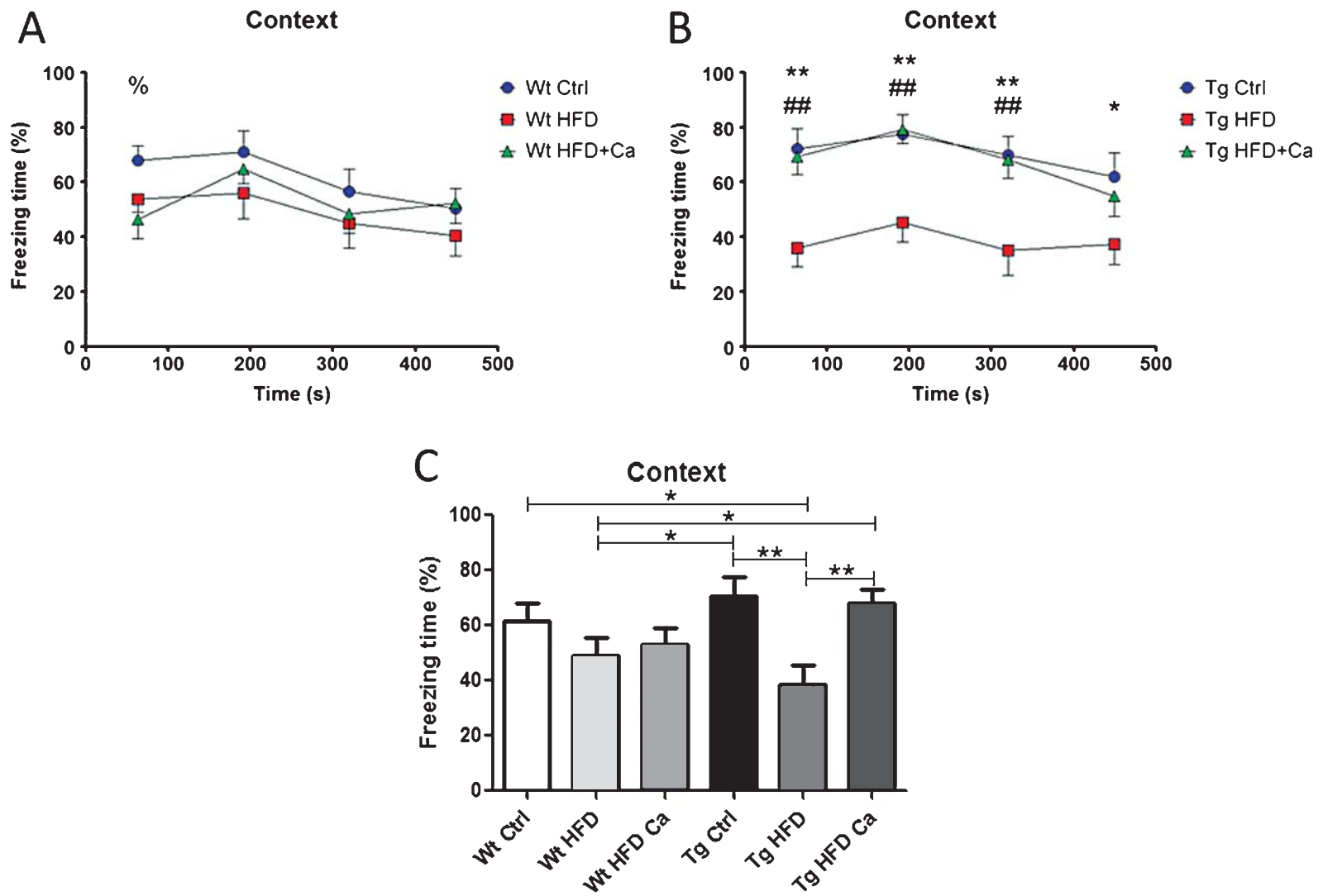

Fig. 4. Contextual memory impairment in transgenic young mice fed with a high fat diet (HFD) and treated with carnosine. Young transgenic mice ( 6 months old) were fed with a HFD for 2 months and treated with carnosine $(5 \mathrm{mg} /$ day) in their drinking water for the final 4 weeks of the feeding. Animals were then subjected to a fear conditioning test. A) Comparative graph of the freezing time of the animals from all wild-type groups; B) Comparative graph of the freezing time of the animals from all transgenic groups; C) Comparison of the total freezing time of the animals during the total time of the experiment. Bars: SEM; Wt, wild-type; Tg, transgenic; HFD, high fat diet; Ca, carnosine. $n=11$ for the wild-type control group, $n=10$ for the wild-type group fed with HFD, $n=12$ for the wild-type group fed with HFD and treated with carnosine, $n=13$ for the transgenic control group, $n=13$ for the transgenic group fed with HFD, and $n=11$ for the transgenic group fed with a HFD and treated with carnosine. *Comparison between Tg Ctrl and Tg HFD; ${ }^{*}$ Comparison between Tg HFD and Tg HFD+Ca; ${ }^{*},{ }^{\%} p<0.05 ;{ }^{* *}$, \#\# $p<0.01$.

brain sections for differences in the levels of RAGE among groups. As a result, we observed an increase in the expression of RAGE in blood vessels in the CA3 region of the hippocampus of transgenic animals fed with HFD (Fig. 7D), something that was not observed either in wild-type animals fed with a normal diet, nor in transgenic animals fed with HFD and treated with carnosine. Furthermore, we also observed an enlargement of the blood vessels in the CA3 region of the hippocampus of transgenic animals fed with HFD (Fig. 7D, arrows). This enlargement was not observed in transgenic animals fed with HFD and treated with carnosine. Following this experiment, we aimed to evaluate whether oxidative stress could be a factor in the observed results. For this reason, we have stained brain sections for acrolein, a marker of peroxi- dized lipids [23, 24]. As a result, we did not observe a significant difference in the accumulation of acrolein between the transgenic groups (Fig. 7J-L).

As inflammation has been reported to be a cause for cognitive decline, we also stained brain sections of the animals utilized in this study for microglial activation that would give us an insight into the histological changes that might be occurring in this model. As a result, we observed that AD mice fed with HFD caused increased microglial activation in the hippocampus (Fig. 8B), something that was not observed in transgenic animals fed with a normal diet (Fig. 8A) as well as wild-type animals fed with a normal diet and wildtype mice fed with HFD (data not shown). This piece of data suggests that neither AD phenotype nor HFD on their own are capable of causing increased microglial 

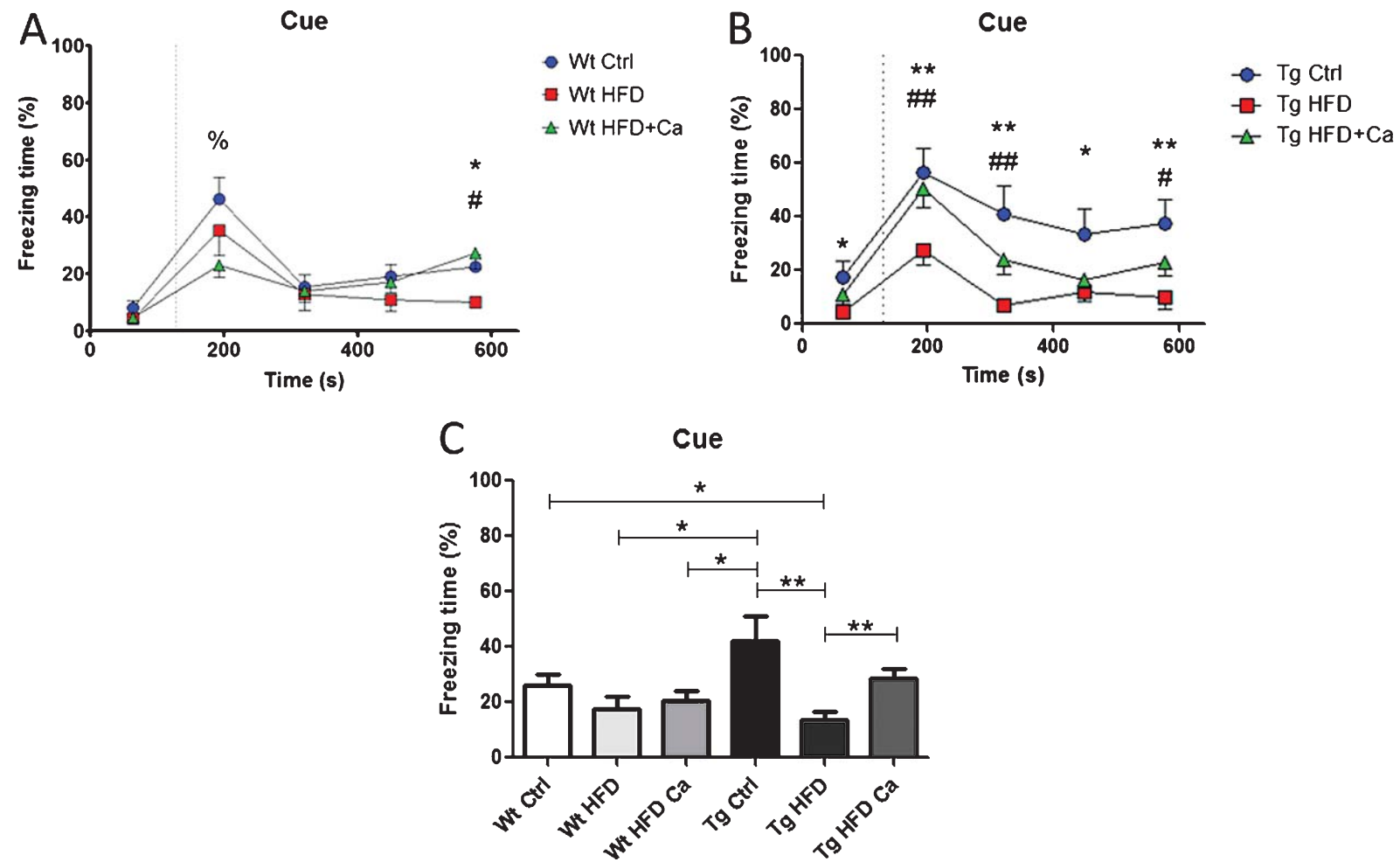

Fig. 5. Cued memory impairment in transgenic young mice fed with a high fat diet (HFD) and treated with carnosine. Young transgenic mice ( 6 months old) were fed with a HFD for 2 months and treated with carnosine $(5 \mathrm{mg} / \mathrm{day}$ ) in their drinking water for the final 4 weeks of the feeding. Animals were then subjected to a fear conditioning test. A) Comparative graph of the freezing time of the animals from all wild-type groups; B) Comparative graph of the freezing time of the animals from all transgenic groups; C) Comparison of the total freezing time of the animals in the wild-type groups during the total time of the experiment; D) Comparison of the total freezing time of the animals in the transgenic groups during the total time of the experiment. For the purposes of this experiment, only time points after the aural cue were used to calculate the total freezing time. Bars: SEM; Wt, wild-type; Tg, transgenic; HFD, high fat diet; Ca, carnosine. $n=11$ for the wild-type control group, $n=10$ for the wild-type group fed with HFD, $n=12$ for the wild-type group fed with HFD and treated with carnosine, $n=13$ for the transgenic control group, $n=13$ for the transgenic group fed with HFD and $n=11$ for the transgenic group fed with a HFD and treated with carnosine.; *Comparison between Tg Ctrl and Tg HFD; ${ }^{\#}$ Comparison between Tg HFD and Tg HFD+Ca; ${ }^{\%}$ Comparison between Tg Ctrl and Tg HFD+Ca. ${ }^{*},{ }^{\#,},{ }^{\%} p<0.05,{ }^{* *},{ }^{\# \#} p<0.01$.

activation, both being required for the occurrence of this phenomenon.

Surprisingly, treatment with carnosine proved effective in preventing this increased microglial activation (Fig. 8C), suggesting that carnosine might interfere with inflammatory pathways. Taken as a whole, these results suggest that treatment with carnosine prevents cognitive decline by preventing an increase in the expression of RAGE and microglial activation.

\section{DISCUSSION}

In summary, the present research has demonstrated that changes in cognitive function were seen between AD transgenic mice fed with HFD and AD transgenic mice fed with normal diet, and amelioration of these detrimental changes with carnosine in the AD mice fed with HFD. However, treatment with carnosine was not capable of preventing or altering the deposition of $A \beta$ in this model. These results suggest that changes in cognitive function are not solely linked to $A \beta$ deposition, but rather caused by the combination of $A \beta$ deposition and something else, possibly an increased expression of RAGE, alongside cerebrovascular alterations and microglial activation.

Through this study we arrived at results that suggest that when the genetic background for $\mathrm{AD}$ is present, obesity per se is not necessarily a main cause for the onset of the cognitive deficits seen in AD mice, as obesity itself had no effect on the cognitive performance of wild-type mice. As transgenic animals treated with carnosine displayed normal cognitive performance, yet remained in the same weight range as their cognitively 


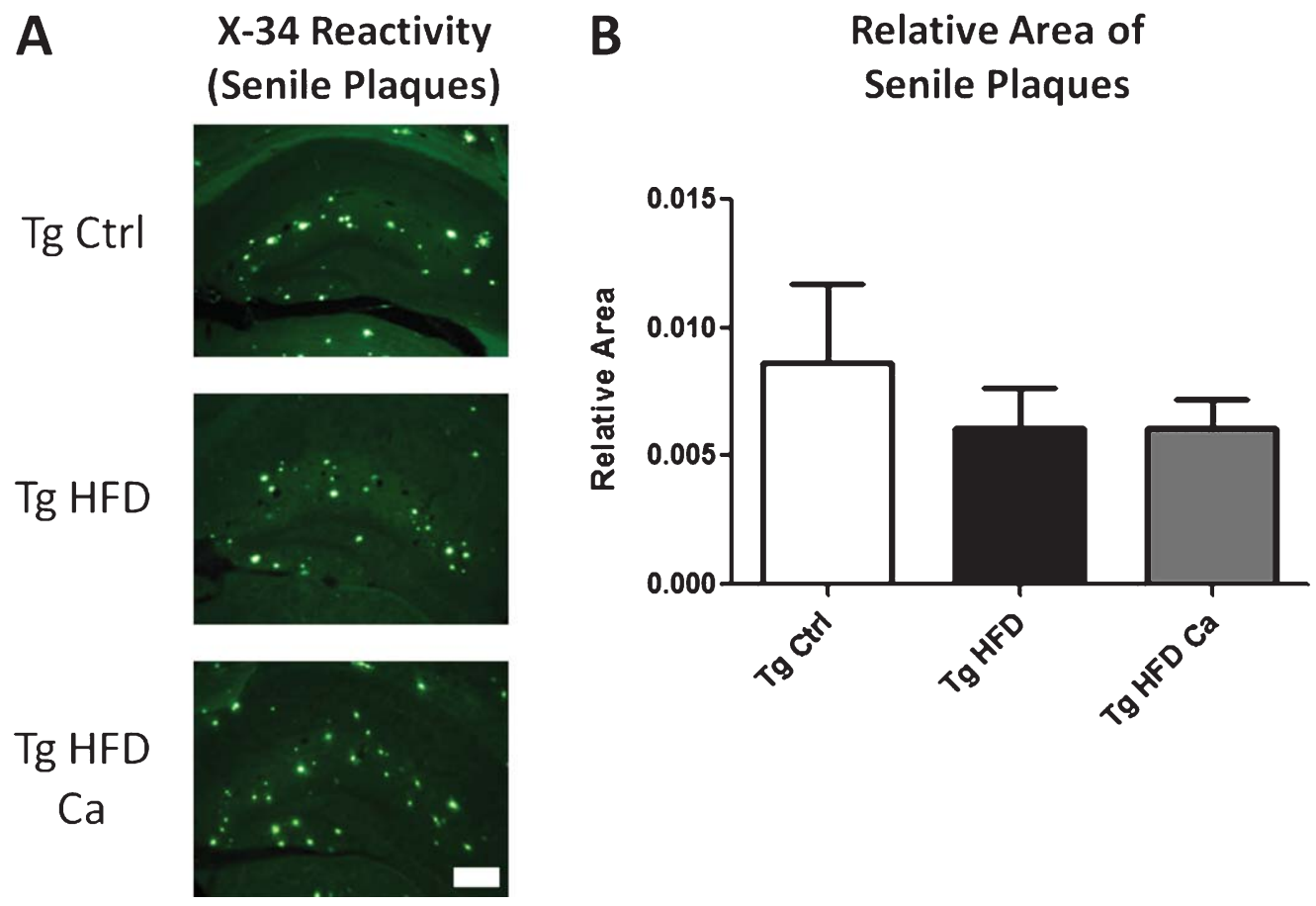

Fig. 6. Histochemical analysis. $24 \mathrm{~h}$ after the end of the cognitive experiments brain samples were obtained and slices for analysis were prepared. Brain sections were stained with X-34, a selective fluorescent dye for senile plaques, and observed in a fluorescence microscope. Photographs of the stained samples were obtained, and the area covered by aggregates stained by X-34 was calculated by a custom ImageJ software plugin. Senile plaques were observed in all groups, and no significant differences in the area covered by senile plaques were observed between the groups. $n=8$ for all groups, values are taken as an average of the reactivity of 3 slices per animal. A) Photographs of the samples. X-34 reactivity is show as bright green spots. Bar: $250 \mu \mathrm{m}$. B) Relative area covered by senile plaques. No differences were observed among groups. Bars: SEM.

impaired littermates, the data herein presented suggests that if those genetic risk factors are present, obesity might then be the trigger, but not the main effector in a cascade of events that culminates in the decay of cognitive functions. This fact is further attested by the finding of increased expression of RAGE and increased microglial activation in transgenic animals fed with HFD, indicating that there might be some intricate relationship between the deposition of the $A \beta$ and the responses evoked by obesity, that synergistically evoke elevated oxidative responses in the brain of transgenic mice fed with HFD. Likewise, feeding with HFD caused significant changes in the blood vessels in the hippocampus, suggesting that the underlying causes for cognitive decline also are capable of causing alterations in the cerebrovascular structure. These pieces of data suggest that while $A \beta$ is a key factor in the etiology of $\mathrm{AD}$, microglial activation, cerebrovascular alterations, and increases in both AGE and RAGE levels could be also largely responsible for cognitive decline, at least in the present model.
AGEs are markers of carbonyl stress that accumulate due to an increased level of sugars. Their formation is irreversible, and their accumulation has been shown to lead to protein deposition and amyloidosis [25]. AGEs usually exert an effect on the brain through RAGE. RAGE has been implicated in the mediation of $A \beta$ toxicity by binding to this peptide and transporting it across the blood-brain barrier, leading to neuroinflammation and neuronal dysfunction. Studies have also shown an increased expression of RAGE in cerebral microvessels in animal models of $\mathrm{AD}$, a result that we have managed to replicate [26, 27]. An increase in the expression of RAGE has been observed in a model of formation of AGEs in vivo induced by methylglyoxal, a reactive aldehyde formed in glucose oxidation or lipid peroxidation [28]. The same study also showed that an increase formation of AGEs is capable of causing endothelial dysfunction, and as such the AGE-RAGE system is a possible cause for the vascular abnormalities observed in our study. We however, did not observe changes in lipid peroxidation, as assessed by 

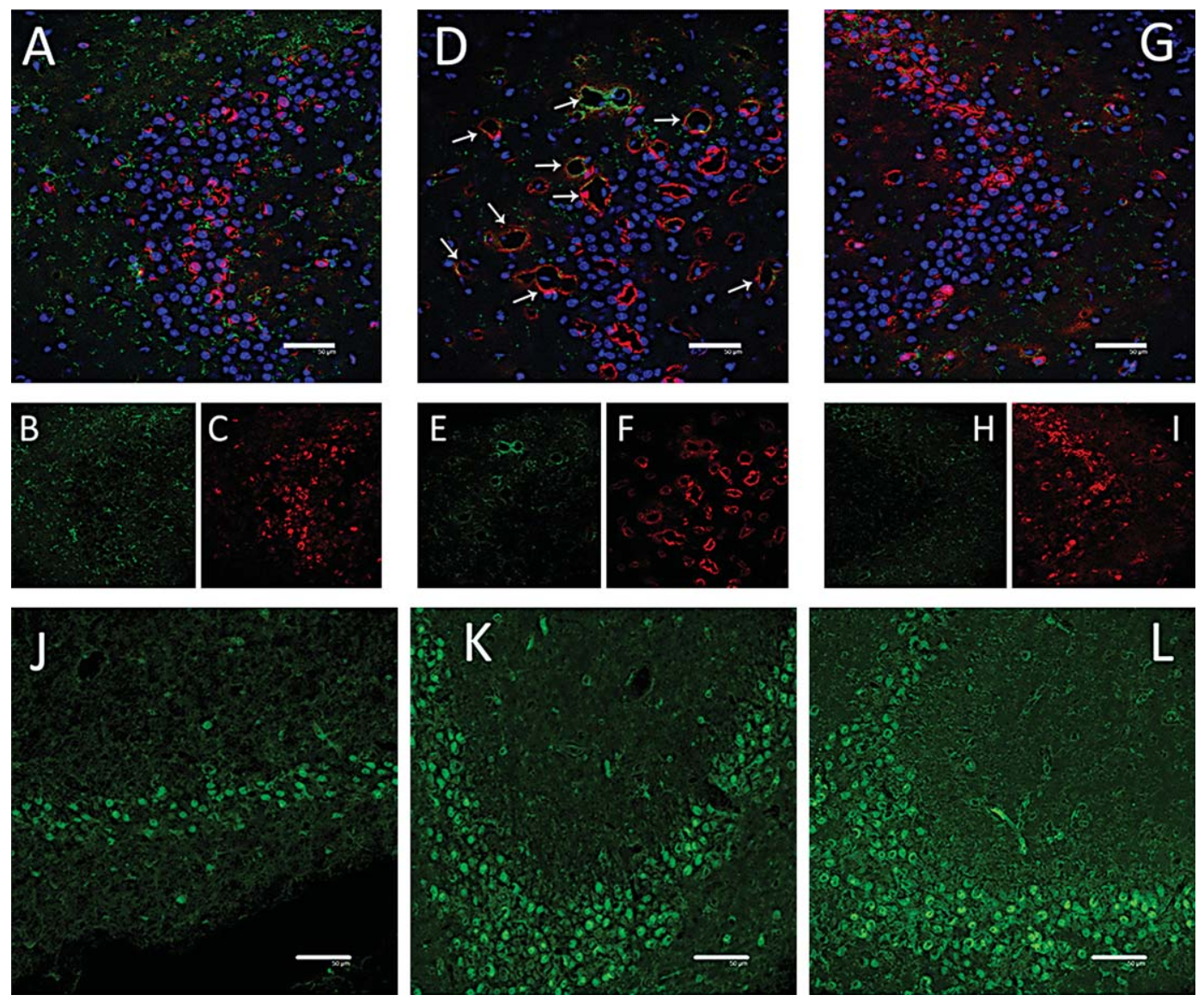

Fig. 7. Immunohistochemical analysis. $24 \mathrm{~h}$ after the end of the cognitive experiments brain samples were obtained and slices for analysis were prepared. Brain sections were preincubated with DAPI (marker of cell nuclei) and primary antibodies for cluster of differentiation 31 (CD-31; marker of blood vessels), receptor for advanced glycation end-products (RAGE), and acrolein (marker of lipid peroxidation). A significant increase in the expression of RAGE in the blood vessels of the CA3 region of the hippocampus was observed in the transgenic animals fed with a high fat diet (HFD). This increased expression was accompanied by an apparent dilation of the blood vessels. These particularities were not observed in the transgenic animals fed with HFD and treated with carnosine. No differences in the accumulation of acrolein were seen between the groups. A-C) CA3 region of the hippocampus of a wild-type animal fed with a regular diet. D-F) CA3 region of the hippocampus of a transgenic animal fed with HFD. Arrows indicate dilated blood vessels with increased expression of RAGE. G-I) CA3 region of the hippocampus of a transgenic animal fed with HFD and treated with carnosine. Merge (A,D,G); RAGE (B,E,H); CD-31 (C,F,I) Blue, DAPI; Bar: 50 $\mu$ m. J) $\mathrm{CA} 3$ region of the hippocampus of a transgenic animal fed with a regular diet. K) CA3 region of the hippocampus of a transgenic animal fed with a HFD. L) CA3 region of the hippocampus of a transgenic animal fed with a HFD and treated with carnosine. Green, acrolein. Bar: $50 \mu \mathrm{m}$.

acrolein immunostaining [23, 24], indicating that lipid peroxidation might not be necessary for activating the AGE-RAGE system in our model.

These previous studies, when taken in conjunction with the data presented herein, suggests that the upregulation of RAGE is directly related to cognitive decline in animal models of AD. The apparent neuroinflammation and dilation of blood vessels we observed in our experiments corroborates findings of other studies, such as that of Takeda et al. [29], that demonstrated that a double transgenic mouse model for type 1 diabetes mellitus and AD displays cerebrovascular inflammation and $\mathrm{A} \beta$ deposition, along with cognitive deficits.

Inflammation might be also one of the factors contributing to the cognitive deficits observed in this model, as we were able to observe increased microglial activation in the hippocampus. That fact alone could account for the differences in contextual memory among the groups utilized for this study. However, we could not observe any differences in microglial activation in the amygdala (unpublished observation), and as such, the reason why differences were observed in the cued memory remain elusive. Nevertheless, while 


\section{A \\ $\mathrm{B}$ \\ C}
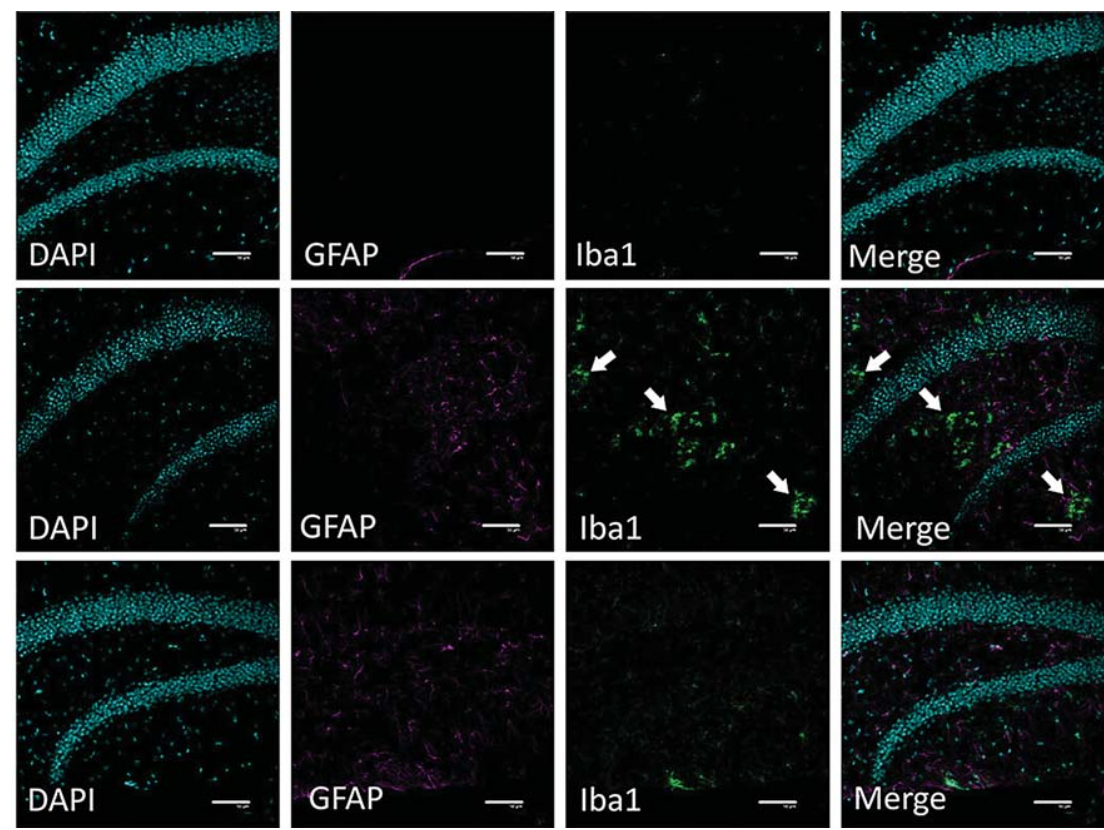

Fig. 8. Immunohistochemical analysis of inflammatory responses in the hippocampus. $24 \mathrm{~h}$ after the end of the cognitive experiments brain samples were obtained and slices for analysis were prepared. Brain sections were preincubated with DAPI (marker of cell nuclei) and primary antibodies for glial fibrillary acidic protein (GFAP, marker of astrocytes) and ionized calcium binding adaptor molecule 1 (Iba1, marker of microglia). Increased expression of Iba1 in clusters was observed in the transgenic animals fed with a high fat diet (HFD) (B, arrows), indicating an increased microglial activation. Such expression was not observed in neither the transgenic animals fed with a normal diet (A), nor in transgenic animals fed with HFD and treated with carnosine (C). Cyan, DAPI; Magenta, GFAP; Green, Iba1; Bar: $50 \mu \mathrm{m}$.

inflammation might not be the initial trigger for neurodegenerative diseases, it has become hard to deny that it plays a significant role in their progression [30]. Experiments with animal models have also shown that an increase in inflammatory mediators such as interleukin $1 \beta$ is capable of inducing impairments in contextual and spatial memory [31]. While it is still unclear how a treatment with carnosine would affect neuroinflammation, our results suggest that it might modulate not inflammation itself, but instead a set of other factors that might lead to inflammation.

Among the putative risk factors for the early onset of $\mathrm{AD}$, for the present research we focused on the induction of a metabolic syndrome, a condition that arises from obesity and encompasses hypercholesterolemia, hyperglycemia, and a systemic resistance to insulin. A metabolic syndrome is taken to be one of the first indicators of the etiology of T2DM, and has also been implicated in the progression of dementia and AD [32, 33]. Likewise, patients affected by T2DM have been found to display a roughly two-fold risk of developing AD later in life, a fact that has since been elaborated on by in extensive reviews such as the ones by Li et al. [34] and Sims-Robinson et al. [33]. Developing on this theoretical and experimental background, we aimed to reproduce this type of risk factor in our animal model by feeding wild-type and $\mathrm{AD}$ model transgenic mice with a HFD for 2 months, a period we deemed to be of sufficient length to evoke a metabolic syndrome in mice, and the subsequent pathological symptoms of AD. We can thus propose a model for explaining why $\mathrm{T} 2 \mathrm{DM}$ is a risk factor for $\mathrm{AD}$, as a synergistic interaction of the complications seen in T2DM and accumulation of the $A \beta$ peptide might be capable of causing cerebrovascular damage that would in turn lead to AD.

Given that the formation of AGEs seems to be a major effector in the development of cognitive deficits, it is highly possible that a treatment with anti-glycating agents would be effective in the prevention of this condition. A report [35] has shown that carnosine is stable in the serum and is available up to $60 \mathrm{~min}$ after dissolution in rats, indicating that it might be a viable therapeutic candidate. The successful prevention of cognitive decline achieved by a 6 -week treatment with carnosine reinforces that notion, and opens many possibilities for future research. In conclusion, we have observed that cognitive changes in this model seem to not be dependent only on $A \beta$, but also on other factors, such as increases in RAGE expression, cerebrovascular abnormalities and neuroinflammation. 


\section{ACKNOWLEDGMENTS}

This study was supported by the Ministry of Education, Culture, Sports, Science and Technology of the Japanese Government (Monbukagakusho - MEXT) as grants to B.H. (Government scholarship), N.K. (24770038) and T.H. (24658117).

Authors' disclosures available online (http://www.jalz.com/disclosures/view.php?id=1524).

\section{REFERENCES}

[1] Jorm AF, Jolley D (1998) The incidence of dementia: A metaanalysis. Neurology 51, 728-733.

[2] Terry RD (2006) Alzheimer's disease and the aging brain. J Geriatr Psychiatry Neurol 19, 125-128.

[3] Biessels GJ, Staekenborg S, Brunner E, Brayne C, Scheltens $P$ (2006) Risk of dementia in diabetes mellitus: A systematic review. Lancet Neurol 5, 64-74.

[4] Profenno LA, Porsteinsson AP, Faraone SV (2010) Metaanalysis of Alzheimer's disease risk with obesity, diabetes, and related disorders. Biol Psychiatry 67, 505-512.

[5] Kohjima M, Sun Y, Chan L (2012) Increased food intake leads to obesity and insulin resistance in the Tg2576 Alzheimer's disease mouse model. Endocrinology 151, 1532-1540.

[6] Reddy VP, Zhu X, Perry G, Smith MA (2009) Oxidative stress in diabetes and Alzheimer's disease. J Alzheimers Dis 16, 763-774.

[7] Nunomura A, Perry G, Aliev G, Hirai K, Takeda A, Balraj EK, Jones PK, Ghanbari H, Wataya T, Shimohama S, Chiba S, Atwood CS, Petersen RB, Smith MA (2001) Oxidative damage is the earliest event in Alzheimer disease. J Neuropathol Exp Neurol 60, 759-767.

[8] Tozuka Y, Wada E, Wada K (2009) Diet-induced obesity in female mice leads to peroxidized lipid accumulations and impairment of hippocampal neurogenesis during the early life of their offspring. FASEB $J \mathbf{2 3}, 1920-1934$.

[9] Cornelli U (2010) Treatment of Alzheimer's disease with a cholinesterase inhibitor combined with antioxidants. Neurodegener Dis 7, 192-202.

[10] Hipkiss AR (2007) Could carnosine or related structures suppress Alzheimer's disease? J Alzheimers Dis 11, 229-240.

[11] Boldyrev AA, Stvolinsky SL, Fedorova TN, Suslina ZA (2010) Carnosine as a natural antioxidant and geroprotector: From molecular mechanisms to clinical trials. Rejuvenation Res 13, 156-158.

[12] Kyriazis M (2010) Anti-ageing potential of carnosine: Approaches toward successful ageing. Drug Discov Today Therap Strategies 7, 45-49.

[13] Dobrota D, Fedorova T, Stvolinsky S, Babusikova E, Likavcanova K, Drgova A, Strapkova A, Boldyrev A (2005) Carnosine protects the brain of rats and Mongolian gerbils against ischemic injury: After-stroke-effect. Neurochem Res 30, 1283-1288.

[14] Rajanikant GK, Zemke D, Senut MC, Frenkel MB, Chen AF, Gupta R, Majid A (2007) Carnosine is neuroprotective against permanent focal ischemia in mice. Stroke 38, 3023-3031.

[15] Min J, Senut MC, Rajanikant K, Greenberg E, Bandagi R, Zemke D, Mousa A, Kassab M, Farooq MU, Gupta R, Majid A (2008) differential neuroprotective effects of carnosine, anserine, and n-acetyl carnosine against permanent focal ischemia. J Neurosci Res 86, 2984-2991.
[16] Shen Y, He P, Fan YY, Zhang JX, Yan HJ, Hu WW, Ohtsu $\mathrm{H}$, Chen Z (2010) Carnosine protects against permanent cerebral ischemia in histidine decarboxylase knockout mice by reducing glutamate excitotoxicity. Free Radic Biol Med 48, 727-735.

[17] Boldyrev A, Koudinev A, Berezov T, Carpenter DO (2004) Amyloid $\beta$ induced cell death is independent of free radicals. J Alzheimers Dis 6, 633-638.

[18] Price DL, Rhett PM, Thorpe SR, Baynes JW (2001) Chelating activity of advanced glycation end-product inhibitors. J Biol Chem 52, 48967-48972.

[19] Sourris KC, Forbes JM, Cooper ME (2008) Therapeutic interruption of advanced glycation in diabetic nephropathy. Ann N $Y$ Acad Sci 1126, 101-106.

[20] Jankowsky JL, Slunt HH, Ratoviski T, Jenkins NA, Copeland NG, Borchelt DR (2001) Co-expression of multiple transgenes in mouse CNS: A comparison of strategies. Biomol Eng 17, 157-165.

[21] Corcoran KA, Lu Y, Turner RS, Maren S (2002) Overexpression of hAPPswe impairs rewarded alternation and contextual fear conditioning in a transgenic mouse model of Alzheimer's disease. Learn Mem 9, 243-252.

[22] Ikonomovic MD, Abrahamson EE, Isanski BA, Debnath ML, Mathis CA, DeKosky ST, Klunk WE (2006) X-34 labeling of abnormal protein aggregates during the progression of Alzheimer's disease. Meth Enzymol 412, 123-144.

[23] Uchida K, Kanematsu M, Sakai K, Matsuda T, Hattori N, Mizuno Y, Suzuki D, Miyata T, Noguchi N, Niki E, Osawa T (1998) Protein-bound acrolein: Potential markers for oxidative stress. Proc Natl Acad Sci U S A 95, 4882-4887.

[24] Stevens JF, Maier CS (2008) Acrolein: Sources, metabolism, and biomolecular interactions relevant to human health and disease. Mol Nutr Food Res 52, 7-25.

[25] Krautwald M, Münch G (2010) Advanced glycation end products as biomarkers and gerontotoxins - A basis to explore methylglyoxal-lowering agents for Alzheimer's disease? Exp Gerontol 45, 744-751.

[26] Yan SD, Chen X, Fu J, Chen M, Zhu H, Roher A, Slattery T, Zhao L, Nagashima M, Morser J, Migheli A, Nawroth P, Stern D, Schmidt AM (1996) RAGE and amyloid- $\beta$ peptide neurotoxicity in Alzheimer's disease. Nature 382, 685-691.

[27] Deane R, Yan SD, Submamaryan RK, LaRue B, Jovanovic S, Hogg E, Welch D, Manness L, Lin C, Yu J, Zhu H, Ghiso J, Frangione B, Stern A, Schmidt AM, Armstrong DL, Arnold B, Liliensiek B, Nawroth P, Hofman F, Kindy M, Stern D, Zlokovic B (2003) RAGE mediates amyloid- $\beta$ peptide transport across the blood-brain barrier and accumulation in brain. Nat Med 9, 907-913.

[28] Sena CM, Matafome P, Crisóstomo J, Rodrigues L, Fernandes R, Pereira P, Seiça RM (2012) Methylglyoxal promotes oxidative stress and endothelial dysfunction. Pharmacol Res 65, 497-506.

[29] Takeda S, Sato N, Uchio-Yamada K, Sawada K, Kunieda T, Takeuchi D, Kurinami H, Shinohara M, Rakugi H, Morishita R (2010) Diabetes-accelerated memory dysfunction via cerebrovascular inflammation and $\mathrm{A} \beta$ deposition in an Alzheimer mouse model with diabetes. Proc Natl Acad Sci U S A 107, 7036-7041.

[30] Glass CK, Saijo K, Winner B, Marchetto MC, Gage FH (2010) Mechanisms underlying inflammation in neurodegeneration. Cell 140, 918-934.

[31] Hein MA, Stasko MR, Matousek SB, Scott-McKean JJ, Maier SF, Olschowka JA, Costa ACS, O'Banion MK (2010) Sustained hippocampal IL-1beta overexpression impairs 
contextual and spatial memory in transgenic mice. Brain Behav Immunity 24, 243-253.

[32] de La Monte S, Wands JR (2008) Alzheimer's disease is type 3 diabetes - evidence reviewed. J Diabetes Sci Technol 2, 1101-1113.

[33] Sims-Robinson C, Kim B, Rosko A, Feldman EL (2010) How does diabetes accelerate Alzheimer disease pathology. Nat Rev Neurol 6, 551-559.
[34] Li L, Hölscher C (2007) Common pathological processes in Alzheimer disease and type 2 diabetes: A review. Brain Res Rev 56, 384-402.

[35] Yeum KJ, Orioli M, Regazzoni L, Carini M, Rasmussen H, Russell RM, Aldini G (2010) Profiling histidine dipeptides in plasma and urine after ingesting beef, chicken or chicken broth in humans. Amino Acids 38, 847-858. 